\title{
Effect of Interferential Therapy over Ultrasound Therapy with Common Protocol of Manual Therapy in Grade - II Frozen Shoulder
}

\author{
Ekisha Gaba, Jasobanta Sethi and Mona Bhardwaj
}

\begin{abstract}
Aim: The aim of the study was to compare the effect of interferential therapy over ultrasound therapy with common protocol of manual therapy in grade -II frozen shoulder. Method: Twenty patients in the age range 35- 65 years having frozen shoulder was selected in the study. Subjects were selected randomly and divided into two groups. One group received interferential therapy, hot pack, shoulder wheel and manual mobilization and other group received ultrasound therapy, hot pack, shoulder wheel and manual mobilization. The regime was taken for two weeks. Patients received treatment every day for two weeks. The outcome of the post-treatment was measured in terms of PAIN and ROM (flexion, extension and abduction) and SPADI score. ROM and PAIN was measured at the end of every seven days. Results: The result showed a significant difference in group A ROM and mild difference in group B ROM. There is no significant difference in pain and disability with both groups. Interferential therapy showed significant improvement in ROM of flexion, extension and abduction in patients with frozen shoulder. Ultrasound therapy helped to improve the tissue structure of glenohumeral joint. There was no significant difference in pain and disability index of shoulder joint. Both interferential therapy and ultrasound therapy helped to decrease pain and disability. Therefore, for pain management both interferential therapy and ultrasound therapy can be utilized as treatment option whereas for ROM interferential therapy has shown significant improvement in patients with frozen shoulder. Conclusion: For pain management both interferential therapy and ultrasound therapy can be utilized as treatment option whereas for ROM interferential therapy has shown significant improvement in patients with frozen shoulder.
\end{abstract}

\section{Ekisha Gaba}

B.P.T.Intern

Amity Institute of Physiotherapy

Amity University, Noida, Uttar Pradesh

E-mail: iamekigaba@gmail.com

\section{Jasobanta Sethi}

Director and Professor

Amity Institute of Physiotherapy

Amity University, Noida, Uttar Pradesh

E-mail: jsethi@amity.edu

\section{Mona Bhardwaj}

Physiotherapist

Taravati Charitable Health Centre

monabharadwaj89@gmail.com

\section{Introduction}

Frozen shoulder ${ }^{[1]}$ or adhesive capsulitis is a distinct clinical syndrome associated with spontaneous onset of gradual progressive pain and restricted range of motion (Martin et al., 2009 ; Nathaniel
Key Words: Pain, ROM, SPADI

DOI: 10.18376/jesp/2020/v16/i2/157454 


\section{Journal of Exercise Science \& Physiotherapy Vol.16 No.2 (July to December) 2020 ISSN: 0973-2020 (Print) $\mathrm{I}_{2} \mathrm{OR}$ Impact Factor $=6.850 \quad$ ISSN: 2454-6089 (Online)}

1993). Frozen shoulder is reported to affect $2 \%$ to $5 \%$ of general population (Nathaniel 1993), increasing to $10 \%$ to $39 \%$ in patients with diabetes and thyroid disease. Individuals with primary frozen shoulder are commonly between 40 and 65 years of age (Manish Samnani 2004; Wordsworth 1986).Patients having no positive findings in their history, clinical examinations or radiographic review that could explain their pain and decrease in shoulder motion are classified as having idiopathic frozen shoulder. The non-traumatic stiff shoulder is usually limited in all directions but the functionally important movements to the patient are flexion, abduction, medial and lateral rotation (Wordsworth 1986). Limitation in range of motion (ROM) occurs due to structural changes in the peripheral tissues. These changes include shortening of capsuloligamentous or muscle as well as adhesion formation. Treatment of limited ROM and function is geared towards applying tensile force in an effort to cause elongation of the restricting tissues. Clinical observations show that all motions increase significantly when treated with therapeutic activities exercises along with modalities. Although the precise aetiology remains unclear, recent evidence identifies that elevated serum cytokine levels are part of process. The aetiology of frozen shoulder is divided into two types primary and secondary. Primary type is of idiopathic origin. Secondary type is divided into three subcategories- systemic, intrinsic and extrinsic. Systemic type includes- diabetes mellitus, hypothyroidism, hyperthyroidism. Intrinsic type includes- rotator cuff tendinitis, rotator cuff tear, bicep tendinitis and acromio clavicular arthritis. Extrinsic type includes- cardiopulmonary disease, cervical disc prolapsed, CVA, humerus fractures and Parkinson disease. The cause of frozen shoulder remains obscure. The original pathological description suggested an inflammatory condition, but sometimes it was found that there was no significant number of inflammatory cells (Brent 2006). It is suggested that the primary pathology was fibrosis. It is also suggested that the coracohumeral ligament gets contracted. Adhesive capsulitis is classically characterized by three stages. The length of each stage is variable, but typically the first stage i.e. freezing lasts for 3-6 months, the second stage frozen lasts for about 3-18 months and the third stage thawing lasts for about 3 to 6 months (Martin et al., 2009). The first stage is freezing. It is characterized by chronic pain with active and passive ROM. Significant limitation of forward flexion, abduction, internal rotation, external rotation. Examination with patients under anaesthesia: ROM essentially identical to ROM when patient is awake (Martin et al., 2009; Burner and Anthony 1995). The second stage is the progressive stiffness or freezing stage. Pain at rest usually diminishes during this stage. Minimal pain except at the end of ROM. Significant limitation of ROM with rigid end feel (Martin et al., 2009). The second stage is the progressive stiffness or freezing stage. Pain at rest usually diminishes during this stage. Minimal pain except at the end of ROM. Significant limitation of ROM with rigid end feel. There is minimal pain present at this stage (Martin et al., 2009). The diagnosis of frozen shoulder may be suggested by a careful history and physical examination. In general, a global loss of active and passive movement is present. Mainly there is loss of flexion, abduction and external rotation. Conservative management of frozen shoulder- NSAID's, corticosteroid injections, oral steroids. Electrotherapeutic modalities- Moist heat, ultrasound, Interferential therapy. Therapeutic treatment - Active ROM exercises (pulley exercise, shoulder wheel, finger shoulder ladder); Passive ROM exercises (stretching, glides). Manual therapy is a form of passive exercise designed to restore joint play motions of roll, glide and joint separation. It is popular conservative treatment of frozen shoulder syndrome. Manual therapy is considered to be modality of choice for restoring or maintaining normal joint play of the synovial joints in the shoulder complex. Exercise is a popular and, most useful form of therapy for frozen shoulder syndrome. Therapeutic exercise regimes are associated with both increased motion and decreased pain in the treatment of frozen shoulder syndrome. 


\section{Journal of Exercise Science \& Physiotherapy Vol.16 No.2 (July to December) 2020 \\ ISSN: 0973-2020 (Print) I I OR Impact Factor $=6.850 \quad$ ISSN: 2454-6089 (Online)}

\section{Materials and Method}

The study consists of twenty subjects (male $=10$, female $=10)$ in the age group of 35-65 years. The subjects included in the study were clinically diagnosed cases of grade -II frozen shoulder. Study Design- Experimental study with comparative nature. The subjects were at Tarawati charitable hospital. The subjects were included in the study considering the following criteria Inclusion criteria

1. Shoulder pain

2. Frozen shoulder grade-II

\section{Exclusion criteria}

1. Shoulder pain due to post fracture stiffness

2. Rheumatoid arthritis

3. Shoulder pain related to cervical spine

4. Tumours

Universal goniometer was used to measure the range of motion of shoulder flexion, extension, abduction and external rotation. Hot pack was given to the patient in supine lying. Hot pack was put for 10 minutes. Overhead pulley was used to regain motion around the shoulder. Flexion, extension, abduction and external rotation were done by this. Shoulder wheel- the ROM around the shoulder was increased. One arm swinging forward, backward, circling. The height of it was adjusted suitably to the required leverage height of the patient. Finger shoulder ladder was used to regain the ROM around the shoulder. It used mainly for abduction and flexion. The height of it was adjusted leverage height of the patient.

The patients were divided into two groups and allocated randomly in groups with 10 cases each. Group -A was treated with interferential therapy whereas group B with ultrasound therapy. Patients were selected as per the inclusive and exclusive criteria. Dosage of interferential therapy is $4 \mathrm{kz}$ at four pole vector 45 for 20 minutes. Dosage of ultrasound therapy was pulsed and frequency of $1 \mathrm{watt} / \mathrm{cm} 2$.Manual therapy exercise included stretching in all direction with anterior and posterior glides. Range of motion was checked before the treatment and scores were taken at the end of 7 th day and 14th day respectively. The pre and post scores of ranges of motion and pain were compared and calculated.

Group-A ( $\mathrm{n}=10)$ : Hot packs, Interferential therapy, and Manual therapy

Group-B $(\mathrm{n}=10)$ : Hot packs, Ultrasound therapy, and Manual therapy

Intervention

Group -A

Interferential therapy- the dosage was $4 \mathrm{khz}$ at 4 pole vector 45 for $20 \mathrm{~min}$.

Group- B

Ultrasound therapy- dosage is pulsed mode $(1: 1)$ of $1 \mathrm{watt} / \mathrm{cm}^{2}$ for 10 minutes.

The intervention of hot pack, passive mobilization and active exercise were given to both Group A and Group B patients.

Hot pack- instructions were given about the treatment. Patient was in supine line. It was put for 10 minutes.

Passive mobilization- Posterior glide was done to increase flexion and internal rotation. Patient's position was in a supine lying. Glided the humeral head posteriorly by moving the entire arm by bending the knees. Anterior glide was done to increase extension and external rotation. Patient's position is in prone lying. Glide the humeral head in an anterior and slightly medial direction. Bend both the knees so that the entire arm was moved anteriorly.

Active exercises- patients were explained about the following techniques and monitored. Each was repeated for 10 times. 


\section{Journal of Exercise Science \& Physiotherapy Vol.16 No.2 (July to December) 2020 ISSN: 0973-2020 (Print) $\mathrm{I}_{2} \mathrm{OR}$ Impact Factor $=6.850 \quad$ ISSN: 2454-6089 (Online)}

- Codman's pendulum exercises- standing with the trunk flexed at the hips about 90 degrees. The hand hanged loosely downwards in a position between 60 and 90 degrees of flexion or scaption. A pendulum or swinging motion of the arm was initiated by having the patient moved the trunk slightly back and forth. Motions of flexion, extension, abduction, horizontal adduction and circumduction were done.

- Pulley exercises were used to regain motion around the shoulder. Flexion, extension, abduction and external rotation were done. Patient was get positive feedback by this.

- Shoulder wheel exercise- the ROM around the shoulder was increased. In arm swinging forward, backward and circling. The height of it was adjusted to the required leverage height of the patient.

- Finger ladder was used to regain the ROM around the shoulder. It was used mainly for flexion and abduction. The height of it was adjusted suitably to the required leverage height of the patient

\section{Outcome measures}

1. Range of motion- The ROM of shoulder joint was measured for 3 movements using a universal goniometer in the following ways.

1.1. Flexion- Patient position was supine lying. Goniometer alignment- fulcrum of the goniometer was placed close to the acromion process. The proximal arm was aligned with the mid axillary line of the thorax. The distal arm was aligned with the lateral epicondyle of the humerus.

1.2 Extension- Patient position was prone lying. Goniometer alignment- fulcrum of the goniometer was placed to the acromion process. The proximal arm was aligned with the mid axillary line of the thorax. The distal arm was aligned with the lateral epicondyle of the humerus.

1.3 Abduction- Patient position was supine lying. Goniometer alignment- fulcrum of the goniometer was placed close to the acromion process. The proximal arm was aligned with the mid line of the anterior aspect of the sternum. The distal arm was aligned with the shaft of the humerus.

2. Shoulder Pain Disability Index (SPADI)

It was a self-administered questionnaire that consisted of two dimensions, one for the pain and other for functional activities. Intraclass correlation coefficient of SPADI is 0.89. The pain dimension consists of five questions regarding the severity of an individual's pain. Functional activities were assessed with eight questions designed to measure the degree of difficulty an individual has with various activities of daily living that require upper extremity use.

Scoring instructions - To answer the questions, patients placed a mark on a $10 \mathrm{~cm}$ visual analogue scale for each question. Verbal anchors for the pain dimension were 'no pain at all' and 'worst pain imaginable', and those for the functional activities were 'no difficulty' and 'so difficult it required help'. The scores from both dimensions were averaged to derive a total score.

Interpretation of scores

Total pain score: - -.-- $/ 50$ x $100=\%$

(Note: If a person does not answer all questions divide by the total possible score, e.g. if 1 question missed divide by 40)

Total disability score: $\ldots$--.- $/ 80 \times 100=\%$

(Note: If a person does not answer all questions divide by the total possible score, e.g. if 1 question missed divide by 70)

Total SPADI score: $\ldots$-..-. $/ 130 \times 100=\%$

(Note: If a person does not answer all questions divide by the total possible score, e.g. if 1 question missed divide by 120)

The means of the two subscales are averaged to produce a total score ranging from 0 (best) to 100 (worst). 


\section{Journal of Exercise Science \& Physiotherapy Vol.16 No.2 (July to December) 2020 ISSN: 0973-2020 (Print) I I OR Impact Factor $=6.850 \quad$ ISSN: 2454-6089 (Online)}

Minimum Detectable Change $(90 \%$ confidence $)=13$ points

(Change less than this may be attributable to measurement error)

\section{Results}

There were two dependent variables for the analysis - ROM and NPRS. Although all the groups reported reduction in pain, disability and improvement in ROM. Group B demonstrated greater increase in range of motion over a period of two weeks. T-test and F-test were used to analyses the data of all the groups on first and last day. These tests demonstrated a significant difference for pain, disability and shoulder ROM (flexion, extension, abduction) over the period of two weeks. T test and F- test were used to analyses the effect of treatment within groups.

Flexion

Table 1. Comparisons of Flexion Range Pre and Post Treatment

\begin{tabular}{|l|l|l|l|l|}
\hline Variable Flexion (degree) & Day -1 & Day-14 & F value & Significant \\
\hline Group-A & $71 \pm 28.84$ & $140.7 \pm 33.18$ & 0.68 & 0.000 \\
\hline Group-B & $101.8 \pm 39.88$ & $164.7 \pm 16.55$ & 0.01 & 0.000 \\
\hline
\end{tabular}

Table 1 show that there was a significant difference between the flexion ROM of first and last day in group A and group $B(p \leq 0.05)$ which is highly significant. This shows that the treatment was effective within the group analysis as the F-value for group A was 0.68 , for group B was 0.01 which was highly significant. The result shows that ROM was increased significantly in all groups after treatment of 2-weeks. But when effectiveness of the treatment was compared between the groups, then group B only showed significant change in ROM of flexion whereas group A did not show any significant change. The mean value of group A for day 1 before treatment is 71 and for day 14 after treatment is 140.7 whereas for group B for day 1 before treatment mean value was 101.8 and for day 14 after treatment is 164.7 .

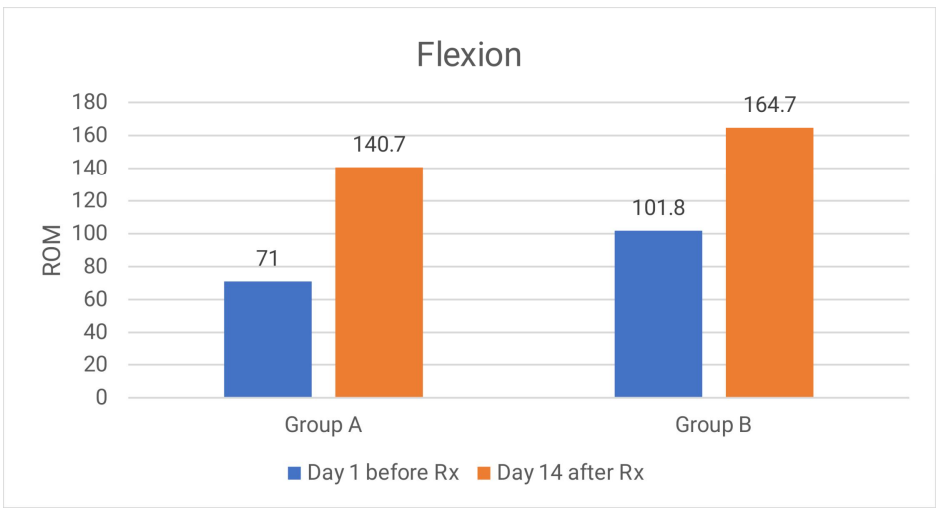

Figure 1. Comparisons of Flexion Range Pre and Post Treatment 
Journal of Exercise Science \& Physiotherapy Vol.16 No.2 (July to December) 2020

ISSN: 0973-2020 (Print) $\quad I_{2}$ OR Impact Factor $=6.850 \quad$ ISSN: 2454-6089 (Online)

Extension

Table 2. Comparisons of Extension Range Pre and Post Treatment

\begin{tabular}{|l|l|l|l|l|}
\hline Variable Extension (degree) & Day -1 & Day-14 & F value & Significant \\
\hline Group-A & $49 \pm 9.94$ & $62.5 \pm 9.78$ & 0.96 & 0.002 \\
\hline Group-B & $56.7 \pm 6.41$ & $60.4 \pm 0.84$ & 1.37 & 0.185 \\
\hline
\end{tabular}

Table 2 shows that there was a significant difference between extension ROM of first and last day in group $A$ as for group $A(p \leq 0.05)$ which is highly significant whereas for group $B(p \geq 0.05)$ which is not significant. This shows that the treatment effective within the group analysis as the F-value for group A was 0.96 for group B was 1.37. The result shows that ROM was increased significantly in all groups after treatment of 2-weeks. But when effectiveness of the treatment was compared between the groups, then group-A showed significant change in ROM of extension than group B. the mean value of group-A for day 1 before treatment is 49 and for day 14 after treatment is 62.5 whereas for group B for day 1 before treatment mean value was 56.7 and for day 14 after treatment is 60.4 .

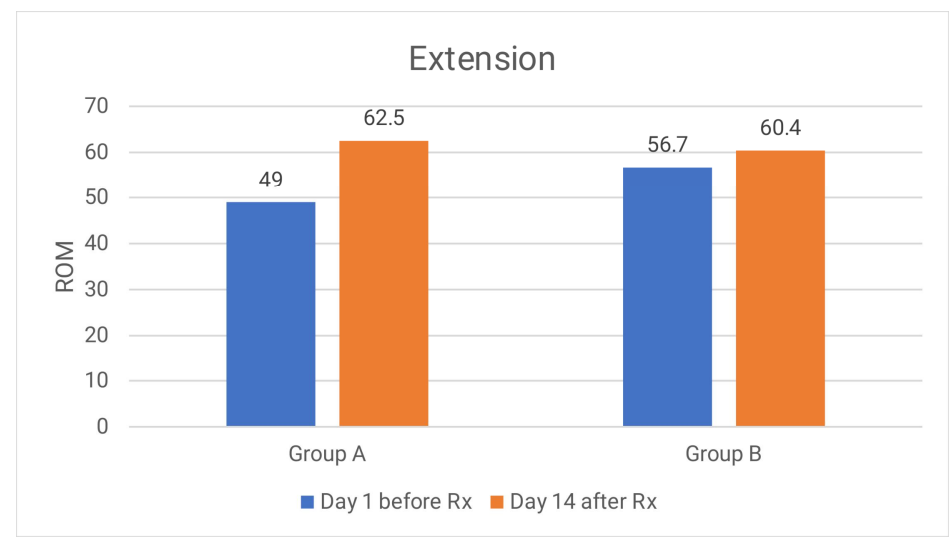

Figure 2. Comparisons of Extension Range Pre and Post Treatment

Abduction

Table 3. Comparisons of Abduction Range Pre and Post Treatment

\begin{tabular}{|l|l|l|l|l|}
\hline Variable Abduction (degree) & Day -1 & Day-14 & F value & Significant \\
\hline Group-A & $77.4 \pm 29.32$ & $170.2 \pm 9.84$ & 0.0003 & -1.94 \\
\hline Group-B & $96.5 \pm 50.39$ & $170.9 \pm 13.55$ & 0.0005 & 0.000 \\
\hline
\end{tabular}




\section{Journal of Exercise Science \& Physiotherapy Vol.16 No.2 (July to December) 2020 \\ ISSN: 0973-2020 (Print) I I OR Impact Factor $=6.850 \quad$ ISSN: 2454-6089 (Online)}

Table 3 show that there is significant difference between the abduction ROM of first and last day in group $A$ and $B(p \leq 0.05)$ which is highly significant. This shows that the treatment effective within the group analysis as the F-value for group A was 0.0003 , for group B was 0.0005 which was highly significant. The resultshow that ROM was increased significantly in all groups after treatment of 2-weeks. But when effectiveness of the treatment was compared between the groups, then group B only showed significant change in ROM of abduction whereas group A did not show any significant change. The mean value of group A for day 1 before treatment is 77.4 and for day 14 after treatment is 170.2 whereas for group B for day 1 before treatment mean value was 96.5 and for day 14 after treatment is 170.9 .

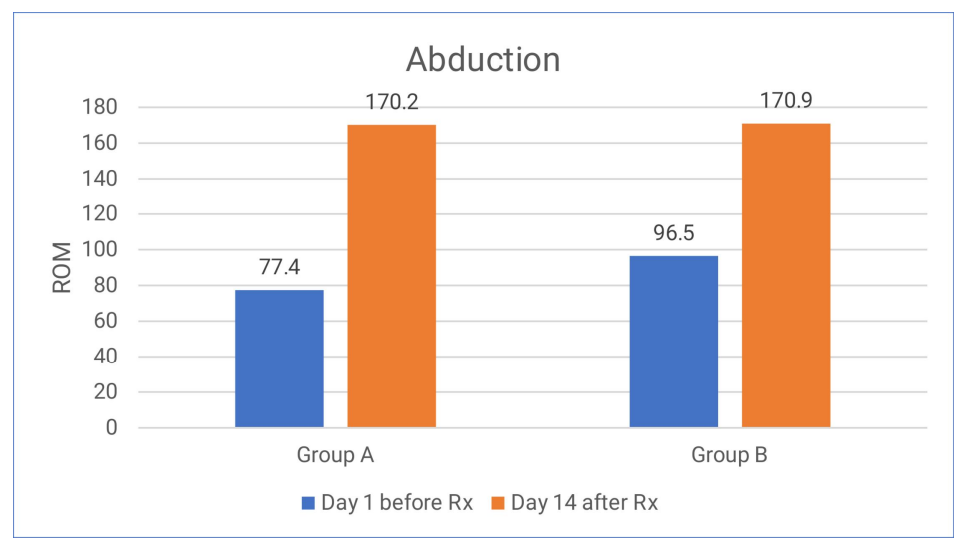

Figure 3.Comparisons of Abduction Range Pre and Post Treatment

NPRS

Table 4. Comparison of NPRS Ranges Pre and Post Treatment

\begin{tabular}{|l|l|l|l|l|}
\hline Variable NPRS & Day -1 & Day-14 & F value & Significant \\
\hline Group-A & $7.1 \pm 2.02$ & $1.3 \pm 1.82$ & 0.76 & -3.72 \\
\hline Group-B & $6.7 \pm 1.49$ & $1.2 \pm 1.39$ & 0.05 & -1.19 \\
\hline
\end{tabular}

Table 4 shows that there was a significant difference between NPRS score for first and last day in group $A$ and $B(p \leq 0.05)$ which is highly significant. This shows that the treatment effective within the group analysis as the F-value for group A was 0.76 , for group B was 0.05 which was highly significant. The results showed that value of NPRS scale decreased significantly in all the groups after treatment of 2-weeks. But when the effectiveness of the treatment is compared between the groups then both the groups did showed drastic improvement. In group A for day 1 the value is 7.1 and for day 14 the value is 1.3 whereas for group B for day 1 the value is 6.7 and for day 14 the value is 1.2 . 


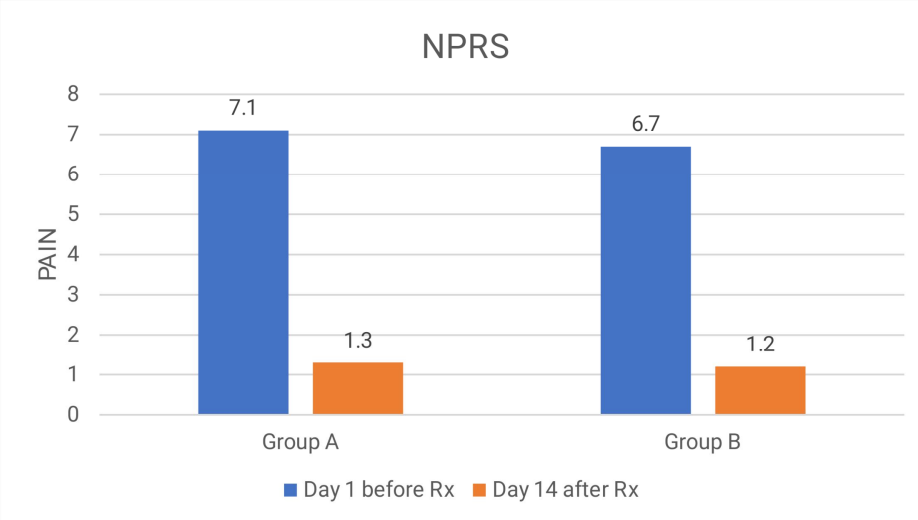

Figure 4.Comparison of NPRS Ranges Pre and Post Treatment

\section{Discussion}

The present study compared the effect of interferential therapy over ultrasound therapy with common protocol of manual therapy in grade -II frozen shoulder. The result of the study on between group analysis, indicate that there is statistically significant difference on improvement of ROM for flexion, extension, abduction for the group who received ultrasound therapy. It also indicates that there is significant difference on improvement of extension and abduction ROM for the group who received interferential therapy along with common exercises. No significant difference was seen in extension ROM. There is also no significant difference in decrease of pain as both groups showed more or less same amount of pain decrease in NPRS score. On the other hand, within group analysis for the group which received interferential therapy revealed a notable improvement in terms of flexion and abduction. There is also notable improvement seen in terms of numeric pain rating scale. The within the group analysis for the group which received ultrasound therapy, revealed a notable improvement in terms of flexion and abduction but not in extension ROM. There is also improvement seen in terms of numeric pain rating scale. Ultrasound therapy gives mechanical vibrations which are high frequency sound waves that are beyond the range of human hearing. It transmits $20 \mathrm{~Hz}$ or $20000 \mathrm{~Hz}$ of frequency. The sound waves will pass more rapidly through material in which molecules are close together. In bone the velocity is $3445 \mathrm{~m} / \mathrm{sec}$, muscle $-1552 \mathrm{~m} / \mathrm{sec}$, cartilage $1665 \mathrm{~m} / \mathrm{sec}$. The production of ultrasound is done using a crystal like quartz which creates a piezo electric effect (the production of emf across a certain substance on being subjected to external pressure). Ultrasound therapy reduces pain, increases local blood flow, decrease joint stiffness, decreases trigger point sensitivity, helps in tissue repair, rebuilding, decreases muscle spasm and pain, reduces inflammation, helps in tissue rebuilding (protein and collagen synthesis).

Interferential therapy gives medium frequency currents associated with lower skin resistance or impedance. The principle of interferential therapy is that it passes two medium frequency alternating currents which intersect each other to produce a low frequency effect. Interferential currents help to decrease pain, give muscle stimulation, increases blood flow and reduces oedema. Adhesions and collagen cross links in the ligament and joint capsule may be the primary extraarticular elements restricting motion. Joint mobilization could increase excursion by breaking these collagen cross links, which forma as result of the decrease in GAG. Joint mobilization could 


\section{Journal of Exercise Science \& Physiotherapy Vol.16 No.2 (July to December) 2020 \\ ISSN: 0973-2020 (Print) I IOR Impact Factor $=6.850 \quad$ ISSN: 2454-6089 (Online)}

certainly affect both joint capsule and the synovial fluid (Nathaniel 1993). The ultrasound treatment given to subjects in group B along with common protocol of exercises resulted in significant gain in flexion, abduction ROM and decrease in NPRS, decrease in joint stiffness within a treatment session when compared to the group A. however group A showed improvement in flexion, abduction \& extension ROM. This provides evidence that ultrasound therapy does alter the mechanics of the joint and increase ROM with common active exercises and joint mobilization were given simultaneously. It has been shown that ultrasound therapy is effective in cases of frozen shoulder than interferential therapy. It will give much more effect on tissue levels than interferential therapy which works primarily on superficial layer to alleviate pain. It will give much more effect if both the therapies are given together. Therefore, when the patient is experiencing pain and loss of ROM ultrasound therapy is required.

\section{Conclusion}

The ultrasound therapy caused a significant improvement in the increase in ROM flexion and NPRS in patients with frozen shoulder. The interferential therapy caused a significant improvement in extension ROM. There is no significant difference in abduction ROM. Both the therapies were found to be better in their own ways. Therefore, both the therapies can be utilized for treatment of acute pain but for tissue healing and repair ultrasound therapy is better than interferential therapy.

\section{References}

Brent S. Brotzman.2006. Clinical Orthopaedic Rehabilitation C. Mosby.2006. 125-250.

Burner T.D. and Anthony PP.1995. The pathology of Frozen Shoulder. Journal of Bone and Joint Surgery.77(5) September 1995 .667-683.

Manish Samnani.2004.Passive exercise coupled with therapeutic activities- a comprehensive study in management of frozen shoulder. Indian Journal of Occupational Therapy:36(2). August -November 2004.37-40

Martin J Kelly et al.2009.Frozen Shoulder: Evidence and a report of proposed model guiding rehabilitation. JOSPT. 3(2) Feb 2009.135-148.

Nathaniel Grubb.1993. Frozen shoulder Syndrome: a review of literature. JOSPT 18(3). Sept 1993.479-487. 